\title{
Border-ownership-dependent tilt aftereffect in incomplete figures
}

\author{
Tadashi Sugihara* \\ The Zanvyl Krieger Mind/Brain Institute, The Johns Hopkins University, 3400 N. Charles Street, Baltimore, \\ Maryland 21218-2685, USA \\ Yoshihisa Tsuji \\ Department of Computer Science, University of Tsukuba, 1-1-1 Tennodai, Tsukuba 305-8573, Japan
}

Ko Sakai

Institute of Information Sciences and Electronics, University of Tsukuba, 1-1-1 Tennodai, Tsukuba 305-8573, Japan

Received March 23, 2006; revised July 26, 2006; accepted August 8, 2006;
posted August 11, 2006 (Doc. ID 69221); published December 13, 2006

A recent physiological finding of neural coding for border ownership (BO) that defines the direction of a figure with respect to the border has provided a possible basis for figure-ground segregation. To explore the underlying neural mechanisms of BO, we investigated stimulus configurations that activate BO circuitry through psychophysical investigation of the BO-dependent tilt aftereffect (BO-TAE). Specifically, we examined robustness of the border ownership signal by determining whether the BO-TAE is observed when gestalt factors are broken. The results showed significant BO-TAEs even when a global shape was not explicitly given due to the ambiguity of the contour, suggesting a contour-independent mechanism for BO coding. (C) 2006 Optical Society of America

OCIS codes: $330.3790,330.5010,330.5510,330.7310,330.7320$.

\section{INTRODUCTION}

The segregation of a figural region from background is a fundamental process for the visual system serving as a crucial step toward the identification of an object and its location. Gestalt factors, such as proximity, smooth continuity, and completeness, have been known as the features that evoke the grouping of line segments. Although such factors have been considered sufficient for figure-ground segregation in contour-based models, e.g., Ref. 1, their roles in segregation processes have not been clarified. Because the extraction of contours in natural images, which is required for contour-based models, has generally been extremely unsuccessful, e.g., Ref. 2, dependence on contour is unlikely to result in a reliable solution. Furthermore, visual images usually contain many elements that could be potentially grouped together through joint action of gestalt factors. Reliance on contours and their junctions does not enable a robust perception of figure-ground segregation.

A neurophysiological study in the macaque has shown that border assignment arises in the early visual cortex areas. ${ }^{3}$ It was reported that a majority of V2 neurons encode border ownership (BO), i.e., on which side a square stimulus is located relative to the receptive field (RF) of a neuron with respect to its optimal orientation. It has not yet been clarified whether the BO coding depends on gestalt factors such as smooth continuity and contour completeness. However, it appears unrealistic that a single neuron in early visual areas can take into account dis- tant, detailed features of contours with short latency. Rather, simpler characteristics of an image are expected to play a crucial role in the robust perception of BO. Surrounding modulation that is reported in early visual cortices could assist figure-ground segregation. ${ }^{4,5} \mathrm{~A}$ computational model that takes into account surrounding contrast, based on the surrounding modulation, has yielded high consistency and robustness for various stimuli in BO signaling. ${ }^{6}$ Because models based on the surrounding modulation rely neither on completeness nor on smooth continuity, such models are consistent with the argument that gestalt factors might not be crucial for $\mathrm{BO}$ determination.

As a step toward the understanding of neural mechanisms underlying the determination of $\mathrm{BO}$, we psychophysically investigated the robustness of BO coding. Specifically, we examined the components of image fragments that are critical for activation of the $\mathrm{BO}$ signal, by measuring the BO-dependent tilt aftereffect (TAE). TAE is a phenomenon whereby an observer perceives a bar tilted in an opposite orientation to the bar with which the same retinal location has been adapted. von der Heydt et al. ${ }^{7}$ presented for adaptation a tilted vertical edge of a trapezoid near a fixation point and examined whether the amount of TAE depends on which side of the fixation point a test square is presented. TAE was observed only when a trapezoid and a test square were placed on the same side, indicating BO-dependent TAE (BO-TAE). We examined whether the completeness and smooth continu- 
ity of probe stimuli are essential for the activation of the BO signal. Specifically, we measured the amount of the BO-TAE, while the length and orientation of line segments of a probe stimulus were systematically manipulated. Note that we anticipate graded effects of completeness, continuity, and BO. For example, as a gap between lines increases, completeness decreases, and BO could be more ambiguous. Strictly speaking, BO should be bimodal in that it takes either side of the border. However, BO could be ambiguous and continuous even in natural conditions when, for instance, amodal completion or occlusion takes place. The results of the experiments showed significant BO-TAEs even when completeness and smooth continuity were weakened or broken, suggesting a contour-independent mechanism for BO determination. We propose a model for the neural mechanisms underlying BO coding, based on our psychophysical evidence, together with neural selectivity for visual stimuli and a receptive field structure of BO-selective neurons.

\section{METHODS}

We examined the effects of contour completeness and smooth continuity on BO-TAEs using a procedure similar to that employed by von der Heydt et al. ${ }^{7}$ Subjects were adapted to a pair of trapezoids, each of which was formed by four line segments, as shown in the left panel of Fig. 1 (adaptation phase). The size of the trapezoids was $89 \mathrm{~mm}$ (6.3 arc deg in visual angle) in height, $73 \mathrm{~mm}$ (5.2 arc deg) and $96 \mathrm{~mm}$ (6.8 arc deg) in top and bottom width, respectively. The trapezoids were shown alternatively to the right or left side by flipping the trapezoid horizontally with respect to the vertical line near the fixation point. This vertical line proximal to the fixation point was tilted $-15^{\circ}$ (clockwise) when the trapezoid was shown on the right, and $+15^{\circ}$ (counterclockwise) when shown on the left, and the midpoint of this line was positioned at 0.86 arc deg beside the fixation point (adapted location). Because the trapezoid was flipped with respect to the midpoint of the slant edge, the midpoint was always at the adapted location with the direction of the slant alternated in accordance with the position of the trapezoid. The trapezoid was shown for $500 \mathrm{~ms}$ with a blank interval of $100 \mathrm{~ms}$ on each side. In total, subjects were adapted to 80 pairs of the trapezoid, resulting in a total adaptation time of $96 \mathrm{~s}$.

A test phase followed the adaptation with a delay of $1000 \mathrm{~ms}$. The test phase consisted of the presentation of a pair of squares as probe stimuli, followed by four pairs of trapezoids as an additional adaptation during the test phase (in-test adaptation), as shown in the right panel of Fig. 1 (test phase). The squares were shown alternatively to the right or left side by flipping the square horizontally with respect to the adapted location. Each square $(71 \mathrm{~mm} \times 71 \mathrm{~mm} \quad$ corresponding to 5.0 arc deg $\times 5.0$ arc deg) was presented for $200 \mathrm{~ms}$, with a blank interval of $1000 \mathrm{~ms}$. The vertical bar of the square at the adapted location was rotated randomly at each presentation (constant stimuli method). The orientation $\left(\theta_{\text {test }}\right)$ ranged between $+2.0^{\circ}$ and $-2.0^{\circ}$ at intervals of $0.2^{\circ}$. The subjects were asked to judge the side to which the edge of the adapted location appeared tilted (two alternative forced choices), after each presentation of a test stimulus, during an interval of $1000 \mathrm{~ms}$. The in-test adaptation was composed of four pairs of the trapezoids, shown under conditions identical to the first adaptation phase. A pair of probe stimuli followed by the in-test adaptation was presented repeatedly 21 times for each session. This procedure was repeated five times over a few days, which resulted in the presentation of 105 pairs of squares (trials) for each configuration of test stimulus.

To examine the effects of the completeness and smooth continuity of contour on the BO-TAE, we systematically changed the horizontal or vertical edge of the square in the test phase. In Experiment 1, the two horizontal edges of the square were manipulated by changing their length and orientation (Fig. 2). The length of the horizontal bars was altered between 0 and 1 at intervals of 0.2 as the ratio to the original length (i.e., $5.0 \mathrm{arc} \mathrm{deg}$ ). The orientation of the horizontal edges was $0^{\circ}, 15^{\circ}$, or $75^{\circ}$ from the horizontal. In Experiment 2, the vertical edge distal to the fixation point was shortened to 0.6 -fold of the original length and rotated $45^{\circ}$ or $90^{\circ}$ from the vertical. Stimuli were white with a brightness of $40.1 \mathrm{~cd} / \mathrm{m}^{2}$, except for the flash in the attention experiments described later, and

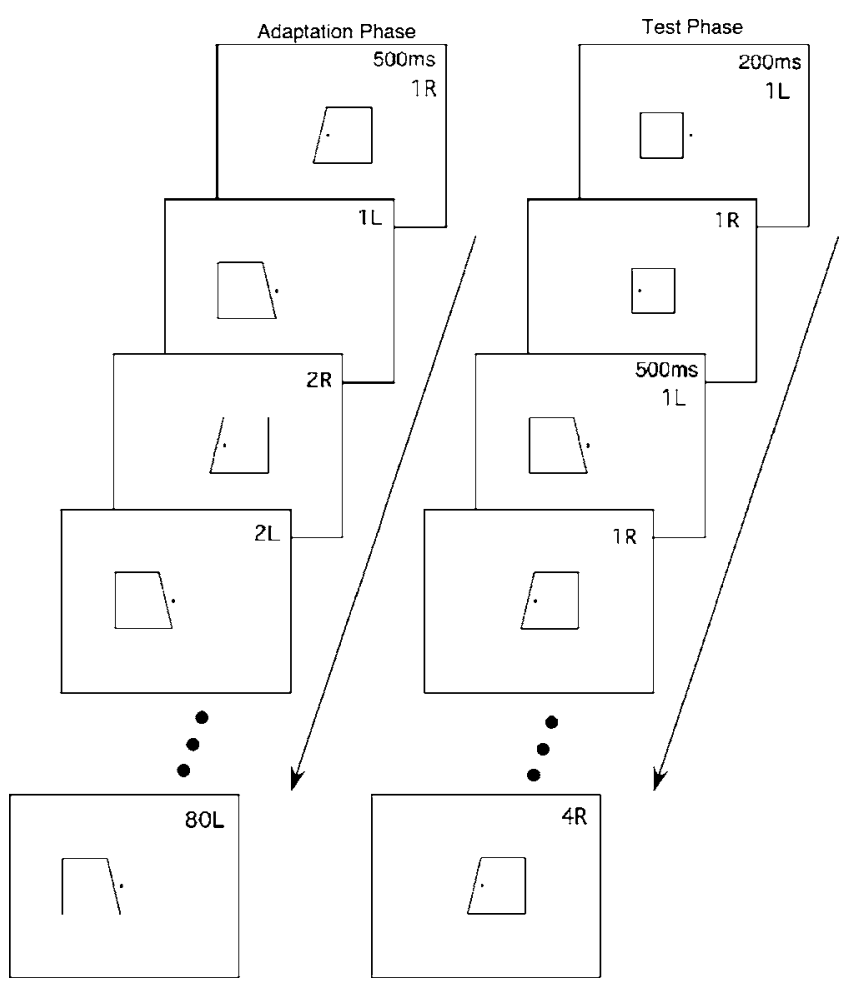

Fig. 1. Experimental procedure. Left: Stimulus configuration during the adaptation phase. Midpoint of the tilted edge $\left(15^{\circ}\right)$ of a trapezoid was situated at 0.86 arc deg beside a fixation aid (indicated by a small black dot). Two trapezoids were shown alternatively for $500 \mathrm{~ms}$ each, and 80 pairs were presented in total (1R, 1L, .., 80L). Right: Stimulus configuration during the test phase. A square (probe stimulus) was shown on the left or right side of the adapted location for $200 \mathrm{~ms}$ each (first $1 \mathrm{~L}$ and $1 \mathrm{R}$ ). The range of orientation of the vertical bar at the adapted location varied randomly within $\pm 2^{\circ}$ at intervals of $0.2^{\circ}$. Subjects were asked to report to which side the vertical bar of the square at the adapted location appeared tilted. In the test phase, adaptation with four pairs of trapezoids $(1 \mathrm{~L}, 1 \mathrm{R}, \ldots, 4 \mathrm{R})$ followed the two probes. 

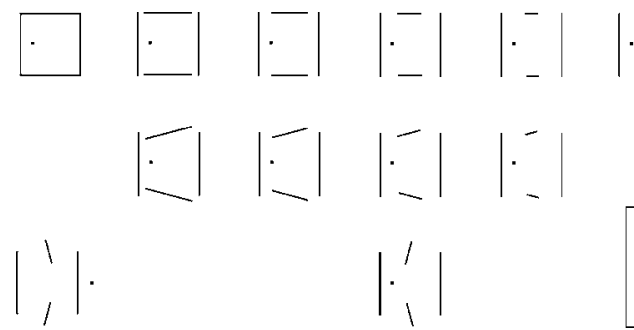

$1,1$.

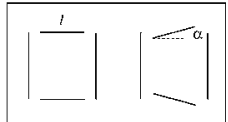

Fig. 2. Probe stimuli for TAE estimation in Experiment 1. Inset on right: Parameter $l$ (left) represents the ratio of the horizontal bar with respect to its original length (5.0 arc deg) and $\alpha$ represents the rotation (right). Left: Two variants of the probe stimulus were formed by either shortening the length of horizontal bars (first row) or by rotating horizontal lines (second and third rows). The original square is shown at the upper-left corner in this panel with a fixation aid (black dot). The length of the horizontal bars was varied between 0.0 and 1.0 with respect to the original with an increment of 0.2 (first and second rows). The orientation of horizontal lines was $0^{\circ}, 15^{\circ}$, or $75^{\circ}$ from horizontal as shown in the first, second, and third row, respectively. All stimuli, except that shown at the bottom-left corner, are those shown to the right side. The stimulus shown to the left side forms the mirror symmetric image to that shown on the right side, with respect to the vertical bar proximal to the fixation aid. An example is shown at the bottom-left corner.

presented on a $21 \mathrm{in.} \mathrm{CRT} \mathrm{monitor} \mathrm{(DELL} \mathrm{Inc.,} \mathrm{Texas)}$ with the spatial resolution of $1600 \times 1200$ at the refresh rate of $75 \mathrm{~Hz}$. The monitor was located in a darkened room with a dim light. Subjects viewed the monitor from a distance of $80 \mathrm{~cm}$ with their heads on a chin rest. All subjects had normal or corrected-to-normal vision. While subject $\mathrm{KS}$ is the author of the present paper, the other subjects were unfamiliar with the aim of the study.

Psychometric functions were fitted by logistic regression analysis to the plots of the response ratio to the counterclockwise perception of the vertical edge of the probe square at a given $\theta_{\text {test }}$. The BO-TAE was defined as the difference between the $50 \%$ threshold of the psychometric functions for each side on which the probe stimulus was presented: $\theta_{\text {estimate }}^{\text {left }}-\theta_{\text {estimate }}^{\text {right }}$. Note that a positive $50 \%$ threshold, $\theta_{\text {estimate}}$ indicates that subjects tended to perceive the vertical edge as tilted to clockwise as a result of adaptation; i.e., the vertical edge must have been tilted physically further counterclockwise to evoke the perception of vertical. The amount of BO-TAE should be positive if there is any significant effect, because $\theta_{\text {estimate }}^{\text {left }}$ should be positive and $\theta_{\text {estimate }}^{\text {right }}$ should be negative by definition. In the case that the visual adaptation in the present study induces negligible aftereffect, the amount of the BO-TAE becomes close to 0 .

Because the BO-TAE is of the order of a few degrees or less, it is, in general, difficult to analyze whether the measured BO-TAE is significantly larger than zero. Therefore, we used the bootstrap method ${ }^{8,9}$ to evaluate the significance of the BO-TAE. In this procedure, the subjects' responses for a particular condition were randomly resampled with replacement followed by the estimation of the BO-TAE with logistic regression analysis. In this way, we minimized the possibility that the significant BO-TAE might be obtained precariously in a specific set of subjects' responses, and we maximized the validity of the amount of the BO-TAE by approximating the sampling distribution of the $\mathrm{BO}-\mathrm{TAE}$ with the resampling proce- dure. For each subject, the resampling procedure was repeated 1000 times by test conditions, yielding 1000 estimated values of the BO-TAE in each test condition. The averaged BO-TAE over subjects and $95 \%$ confidence interval were estimated by this procedure. If the $95 \%$ confidence interval estimated by the bootstrap distribution of the BO-TAE does not include zero, it means that the obtained BO-TAE is significantly positive; therefore, we consider the BO-TAE significant. Data analysis was performed using the S-PLUS version 6.0 (Insightful Corp., Wash.).

\section{MANIPULATION OF A HORIZONTAL BAR: EXPERIMENT 1}

We quantitatively examined the amount of BO-TAE for stimuli with and without contour completeness, which was manipulated by the length of the horizontal bars. For example, when the horizontal bars were shortened, a gap at each corner of a square increased, and the degree of closure was thus diminished. Note that we assume graded completeness; as a gap extends, completeness decreases. In the extreme case, horizontal bars disappeared and only two vertical bars remained. Figure 3(a) shows the BOTAE for three subjects (OM, SK, and WN) as a function of the length of the horizontal bars. The BO-TAE induced by a completed square was $2.0^{\circ}$, as shown at the abscissa of 1.0. The mean BO-TAE and 95\% confidence intervals (bootstrap percentile confidence interval) across subjects in each condition are described in Table 1 . The $95 \%$ confidence intervals are also shown as error bars in Fig. 3(a). The results show that the BO-TAE was significantly larger than 0 at the $95 \%$ confidence level for the entire range of the length of the horizontal bars. Thus, the shortened horizontal bars induced the BO-TAE, and most strikingly, the BO-TAE was present even when the horizontal bars were not shown. These results suggest that the completeness of contour is not required to produce the BO-TAE.

Although contour incompleteness was introduced in these stimuli with short horizontal bars, the remaining line segments or even lack thereof could form subjective contours to complete the square. The rotation of the horizontal bars eliminates this possibility as they disrupt the subjective completion of contours from smooth continuity among the remaining line segments. We investigated the effects of tilted horizontal bars that disrupt the smooth continuity of the contour. Figure 3(b) shows the BO-TAE when the smooth continuity was disrupted by rotating the horizontal bars by $15^{\circ}$ in the test stimuli. The mean and 95\% confidence intervals of the BO-TAE are shown in Table $1\left(\right.$ tilt $\left.=15^{\circ}\right)$, indicating significant BO-TAEs for all conditions. The $95 \%$ confidence intervals are also shown as error bars in Fig. 3(b). We further rotated the horizontal bars to $75^{\circ}$, and obtained significant BO-TAEs, as shown in Table $1\left(\right.$ tilt $\left.=75^{\circ}\right)$, although the amount of TAE for stimuli with a $75^{\circ}$ tilt was smaller than for those with a $15^{\circ}$ tilt.

To further assess the influence of length of the horizontal bars and their orientation on the BO-TAE magnitude, we collapsed the data obtained for the $0^{\circ}$ and $15^{\circ}$ tilt conditions, and carried out a three-way analysis of variance 
(ANOVA) (three-way ANOVA: SuBJECT $\times$ LENGTH $\times$ ORIENTATION). Here, we used the original nonbootstrapped BO-TAE from each subject. LENGTH and ORIENTATION corresponded to the parameters $l$ and $\alpha$ in Fig. 2 . We first made an additive model by sequentially adding the factors. There were significant main effects of SuBJECT and LENGTH ( $p<0.002$ and $p<0.020$, respectively), but no significant main effect of ORIENTATION $(p>0.2)$, suggesting that the BO-TAE was independent of horizontal bar orientation. Next, we dropped the factor ORIENTATION and ran a two-way ANOVA (SUBJECT $\times$ LENGTH) with the consideration of a possible interaction between the two factors. There were main effects of SUBJECT and LENGTH $(p<0.005$ and $p<0.03$, respectively), but there was no effect from interaction $(p>0.40)$. A tendency of slight dependence on the length of the horizontal bar was shown by fitting the continuous univariate response (BOTAE) as a linear function of a single predictor variable, LENGTH. The result of the linear fit showed that the intercept was at $0.97^{\circ}(p<0.0001)$ and the slope was $0.91(p$ $<0.02$ ). Horizontal bars of a square-probe stimulus that were modified either in length or orientation did not abolish the BO-TAE, though there was a general tendency of the magnitude of the BO-TAE to increase as the degree of completeness increased.

\section{MANIPULATION OF A VERTICAL BAR: EXPERIMENT 2}

We found that the BO-TAE does not necessarily require completeness or smooth continuity of the probe stimulus when horizontal bars are shortened or rotated. Next, we examined whether there was any effect of the vertical bar of a probe stimulus on the magnitude of the BO-TAE. We manipulated the orientation of the vertical bar distal to the adapted location while the horizontal bars were unchanged. The lengths of the vertical bars were fixed at the ratio of 0.6 to the original length. The vertical bar was rotated by $45^{\circ}$ and $90^{\circ}$. An additional condition was introduced by completely deleting the vertical bar distal to the adapted location. Figure 4(a) illustrates the configuration of the stimuli.
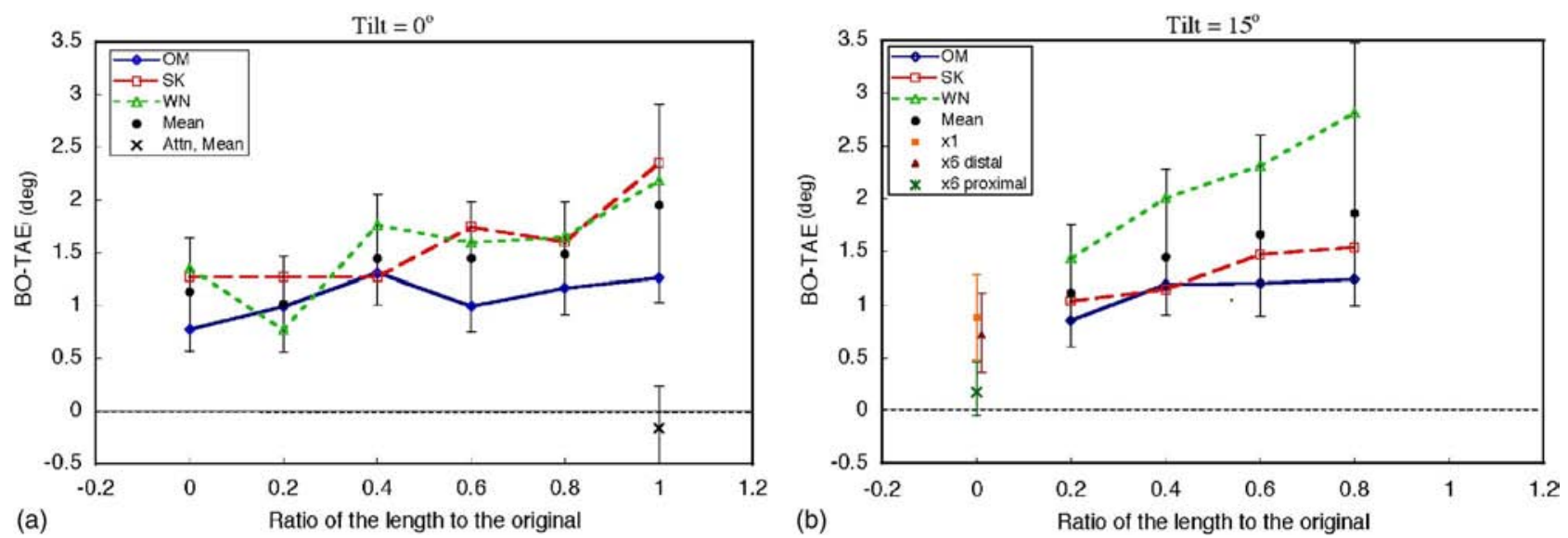

Fig. 3. (Color online) Estimated BO-TAE as a function of the ratio of the horizontal bar length to the original length. Three types of open icons with distinct connecting lines identify the subjects. Solid circles show mean BO-TAE among the three subjects, with error bars indicating corresponding 95\% confidence intervals obtained by the bootstrap method. The orientation of the test horizontal bar was (a) $0^{\circ}$ and (b) $15^{\circ}$. The BO-TAE for a completed square is shown where the abscissa is one. A star icon at the right bottom corner of (a) represents the result for attention experiment (Experiment 3). The mean BO-TAEs among three subjects for the stimuli with narrow edges (circle icon), a wide distal edge (triangle), and a wide proximal edge (star) are shown where the abscissa is $\sim 0$ in panel (b), with error bars indicating corresponding $95 \%$ confidence intervals.

Table 1. Mean and 95\% Confidence Intervals of BO-TAE Estimated by the Bootstrap Method for Tilt $(\alpha)$ of $0^{\circ}, 15^{\circ}$, and $75^{\circ} a$

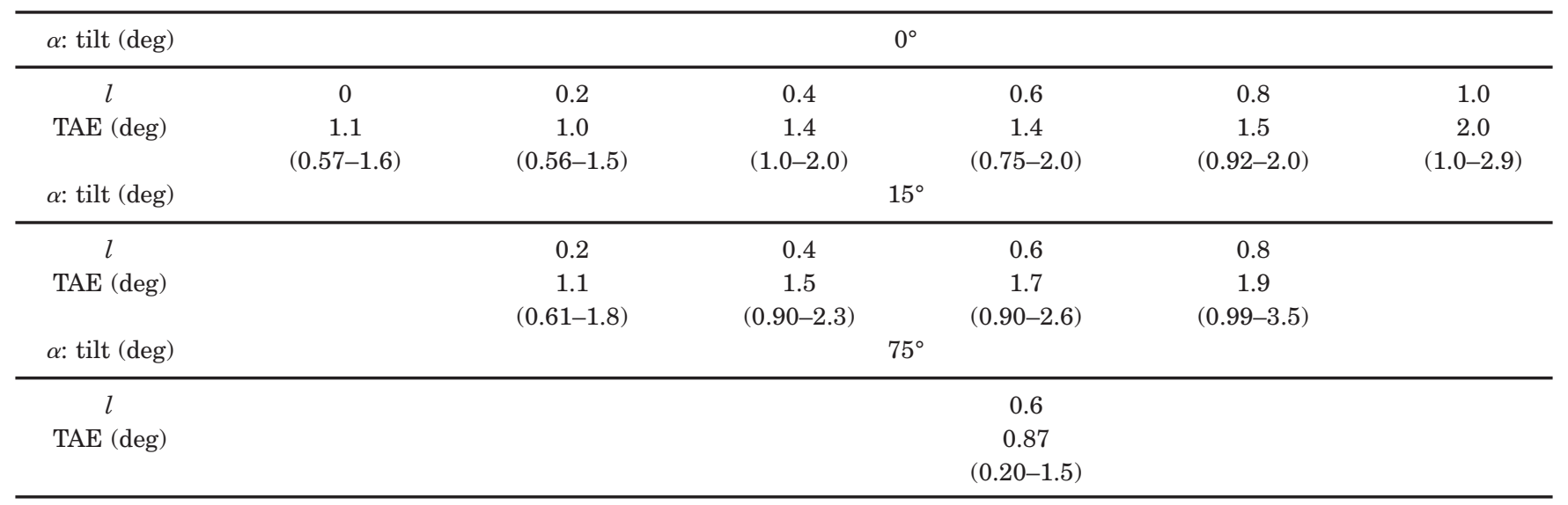

${ }^{a} l$ denotes the ratio of the length of the horizontal bar to the original length. The $95 \%$ confidence intervals are described in parentheses. 


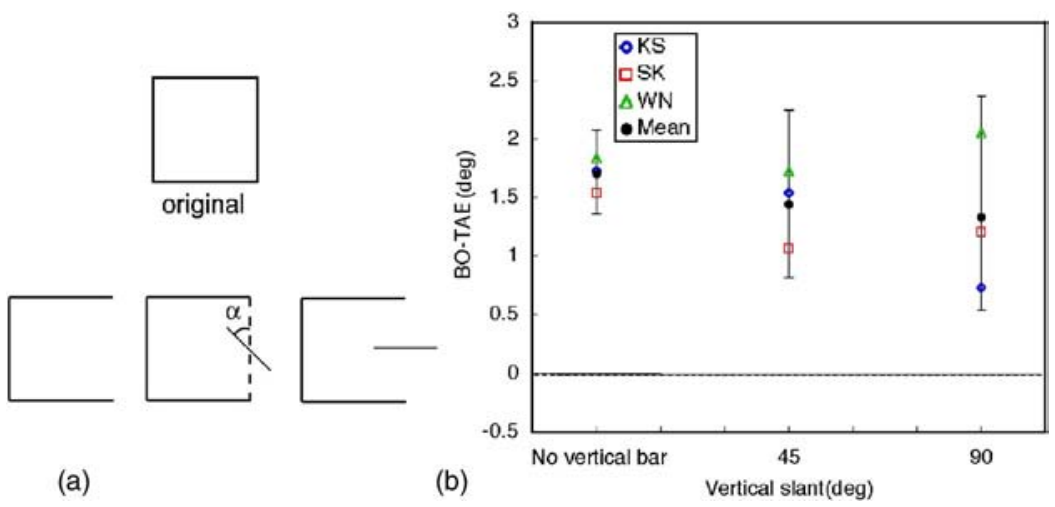

Fig. 4. (Color online) (a) Probe stimuli for the measurement of BO-TAE with variations of a vertical bar (Experiment 2). The ratio of the length of the vertical bar to the original was 0.6. The orientations of the vertical bar $(\alpha)$ were $45^{\circ}$ and $90^{\circ}$. Including a no-vertical-bar condition, there were three conditions in total. (b) Measured BO-TAE as a function of vertical slant. Three types of open icons identify the subjects. Solid circles show mean BO-TAE among the three subjects with error bars indicating corresponding 95\% confidence intervals obtained by the bootstrap method. Note that data at "No vertical bar" indicate the results for the no-vertical-bar condition.

The estimated BO-TAEs for three subjects (KS, SK, and $\mathrm{WN}$ ) are shown in Fig. 4(b). The BO-TAE is observed even when the vertical bar is rotated to break contour completeness and smooth continuity. The magnitude of the BO-TAE for the no-vertical-bar condition was similar to that observed for a completed square. The bootstrapped mean BO-TAEs over three subjects were $1.7^{\circ}, 1.4^{\circ}$, and $1.3^{\circ}$ for the no-vertical bar, $45^{\circ}$ and $90^{\circ}$ tilt, respectively. The corresponding $95 \%$ confidence intervals were $1.4^{\circ}-$ $2.1^{\circ}, 0.82^{\circ}-2.3^{\circ}$, and $0.54^{\circ}-2.4^{\circ}$, respectively, as shown by the error bars in the figure. The two-way ANOVA without replication (additive model) was applied to the nonbootstrapped BO-TAE for individual subjects and revealed no significant main effects of SUBJECT or ORIENTATION ( $p$ $>0.2, p>0.4$, respectively). We conclude that a significant BO-TAE was induced, even when the vertical bar that was located distally to the adapted location was rotated to break completeness and smooth continuity of the probe stimulus.

\section{EFFECTS OF COVERT SPATIAL ATTENTION: EXPERIMENT 3}

The results thus far suggested that inducing the BO-TAE is not dependent on the completeness or smooth continuity of the probe stimulus. One possibility that may have accounted for the BO-TAE in our experiment would be spatially biased covert attention. Although we associate BO between adaptation stimuli and test stimuli, it could be the direction of stimulus presentation with respect to the RF. If the stimulus presentation serves as a cue to draw bottom-up attention, because the direction of the stimulus presentation is common between the adaptation and test, TAE could depend on the direction of attention rather than BO. Neural responses in the monkey area V4 are modulated by the location of attention in the visual field, ${ }^{10,11}$ and, more specifically, the $\mathrm{BO}$ signal in V2 is also modulated, depending on the location of attention. ${ }^{12}$ Therefore, it is important to examine the effects of covert spatial attention on the BO-TAE.

To examine whether bottom-up attention to the presence of the probe stimulus on one of the two sides would result in a BO-TAE, we presented a small bright square
(151.1 $\mathrm{cd} / \mathrm{m}^{2}, 17$ arc min side) for $100 \mathrm{~ms}$ at the location of the vertical line that was distal to the fixation point (i.e., not on the adapted location) without any line segment. The vertical bar at the adapted location with its orientation randomized followed the flash without delay. No other line segment was present simultaneously. A blank interval of $1000 \mathrm{~ms}$ followed the presentation of the vertical line. The test stimulus was composed of the flash, the vertical bar, and the blank interval, which was repeated with the flash flipped on the other side and followed by the in-phase adaptation as identical to the previous experiments. All procedures, except for the test stimulus, were identical to the previous experiments. The bootstrapped estimation of the mean BO-TAE was $-0.15^{\circ}$, and the $95 \%$ confidence interval was $-0.54^{\circ}$ to $0.38^{\circ}$, as plotted by a star icon and its error bar in Fig. 3(a), indicating that the BO-TAE was not significant in this condition. Based on this observation, we conclude that the observed BO-TAE was not due to spatial attention.

\section{DISCUSSION}

We studied the influence of stimulus configuration on the BO-TAE in order to examine the robustness of BO coding in terms of contour completeness and smooth continuity. Results showed that manipulation of the length of the line segments did not abolish the BO-TAE. Even when two horizontal bars were completely deleted, a significant BO-TAE was observed. Our results also showed that rotating either horizontal or vertical bars did not abolish the BO-TAE. The present experiments demonstrated that BO coding is robust even when gestalt factors are suppressed. Note that the BO-TAE cannot be explained by a neural mechanism that only employs neurons selective for orientation of bars. During the adaptation phase, such neurons are adapted to the two orientations of the slant bars of a pair of trapezoids. In fact, this expectation was corroborated by the additional experiment in which single, proximal bars were presented as test stimuli. No significant BO-TAE was observed in this experiment (mean BO-TAE $=-0.066^{\circ}, 95 \%$ confidence interval $=$ $-0.43^{\circ}$ to $0.22^{\circ}$ ). This strongly indicates that the BO-TAE originates from the fatigue of $\mathrm{BO}$-selective cells. 
Simulation studies ${ }^{6,13}$ have shown that most properties of BO-selective cells can be evoked from asymmetric distribution of facilitatory and suppressive surrounding regions reported in early vision. ${ }^{14,15}$ For example, when a stimulus is placed onto a facilitatory region of a cell, it enhances the cell's responses, and therefore the cell behaves as if the contour is owned by the side where a facilitatory region is present. Sakai and Nishimura ${ }^{6}$ predict that this asymmetry is the key for the determination of BO, and cells in any visual area that have this property can achieve BO selectivity. Note that, although their model consists of neurons without dynamics and anatomical plausibility, the spatial nonlinearity of $\mathrm{V} 1$ neurons is reproduced. ${ }^{16,17}$ In their model, even when horizontal bars of the probe stimulus are missing, BO is retained if the vertical bar distal to the adapted location falls into either a facilitatory or suppressive region. Therefore, this theory can account for the presence of BO-TAEs when horizontal bars are completely deleted or extremely slanted.

Recent physiological study has shown that surround modulation can be evoked by stimuli with a combination of the preferred spatial frequency for the classsical receptive field $(\mathrm{CRF})$ and a lower frequency for the outside of the CRF. ${ }^{18}$ This frequency characteristic of the surrounding effect leads to an interesting prediction for a BO-TAE. Because the TAE is a spatial-frequency-dependent phenomenon, ${ }^{19}$ the TAE is evoked only if the width of a line segment shown during the test phase is the same as that shown for adaptation. This constraint should hold true for the BO-TAE; the widths of the proximal line segments should be the same or similar throughout the adaptation and test phases. Therefore, if a proximal edge of a test stimulus is widened with respect to that of adaptation stimuli, we predict that a BO-TAE will not be observed. However, considering the findings that surround modulation persists for a lower frequency range, the width of a distal line segment could be wider to evoke a BO-TAE, if BO is determined mainly by the effect of surrounding modulation. Our preliminary results indicated that this prediction is veridical for a test stimulus without horizontal edges, as shown in Fig. 3(b). We widened either a proximal or a distal edge by a factor of 6 , and observed a significant $\mathrm{BO}-\mathrm{TAE}$ only for the latter case with the distal edge widened (mean $\mathrm{BO}-\mathrm{TAE}=0.72^{\circ}, 95 \%$ confidence interval $=0.36^{\circ}$ to $1.11^{\circ}$ ). Although the width of the bars does not correspond directly to spatial frequency, our preliminary results support that the surround modulation is a basis for BO selectivity.

It should be noted that completeness of the probe stimulus enhanced the magnitude of BO-TAE; as the completeness of the stimulus deteriorated further, the observed BO-TAE became weak, which would suggest the coexistence of influence from Gestalt factors. Given the limited number of conditions of the orientation, our results did not show a significant effect of orientation on BO-TAE. However, as in the effect of length, angular deviation would be expected to contribute to the change in BO-TAE magnitude. Although BO should be bimodal in essence, either left or right in our experiments, the BOTAE exhibits values in between the two, depending on the degree of completeness. This continuous change in BO-
TAE might suggest the continuum and probabilistic nature of BO signaling. BO-selective cells could signal the degree of $\mathrm{BO}$ based on its preference, which appears to be inherent to the model based on surrounding modulation. Alternatively, but not necessarily exclusive to the graded response nature inherent to individual cells, a population of cells might represent probability of BO. Sajda and Baek $^{20}$ have introduced probability of the direction of figure (DOF) based on Bayesian inference, which seems to give a good conceptual basis for figure-ground segregation. It would be interesting to investigate the correspondence of BO-TAE and the probability of DOF.

A number of BO-selective cells should participate in determining the DOF of the entire contours of an object. Several studies have proposed the computational models that take a population of cells into account to determine the DOF along the whole contours of an object. ${ }^{20-22} \mathrm{Al}-$ though these models use relatively simple mechanisms to realize $\mathrm{BO}$ selectivity of single cells, such as detection of convexity, similarity, and proximity of contour segments, local communications among neighborhoods enable the detection of the DOF along the whole contour including occlusion and transparency. These contour-based models appear to be inconsistent with our results, because the BO-TAE is observed even if horizontal bars are completely deleted. However, these population models may account, in part, for the decline in the magnitude of $\mathrm{BO}$ TAE when the completeness of the stimulus is deteriorated. For example, suppose that the mechanism for the determination of the DOF is independent of spatial frequency so that the model takes into account lower frequency components that blur smaller gaps. The model would tend to signal smaller BO-TAE for larger gaps, because only lower frequency channels play a part in the BO determination for larger gaps. Neurophysiological experiments that examine the receptive field structures of BOselective neurons, as well as their behavior as a population, will further advance our knowledge of BO coding and figure-ground segregation. We studied the robustness of BO coding using the tilt-aftereffect phenomenon and demonstrated that this coding works even when visual stimuli break gestalt factors such as contour completeness and smooth continuity.

\section{ACKNOWLEDGMENTS}

This work was supported by a grant-in-aid for scientific research from Japan Society for the Promotion of Science (KAKENHI 13680861, 15500203) and the Brain Science Foundation. We thank S. L. Brincat, D. Dilks, and S. Edelman for improving earlier drafts and for their valuable comments. We also thank N. Aoki for the experimental setup and data collection. We thank M. Nakazono for providing useful suggestions on the statistical analysis with S-PLUS.

Corresponding author Ko Sakai's e-mail address is sakai@cs.tsukuba.ac.jp.

*Present address, Department of Neurobiology and Anatomy, University of Rochester, 601 Elmwood Avenue, Box 603, Rochester, New York 14642. 


\section{REFERENCES}

1. P. Sajda and L. H. Finkel, "Intermediate-level visual representations and the construction of surface perception,” J. Cogn. Neurosci. 7, 267-291 (1995).

2. D. Marr, Vision (Freeman, 1982).

3. H. Zhou, H. S. Friedman, and R. von der Heydt, "Coding of border ownership in monkey visual cortex," J. Neurosci. 20, 6594-6611 (2000).

4. V. A. F. Lamme, "The neurophysiology of figure-ground segregation in primary visual cortex," J. Neurosci. 15, 1605-1615 (1995).

5. S. Kastner, H. C. Nothdurft, and I. N. Pigarev, "Neuronal correlates of pop-out in cat striate cortex," Vision Res. 37, 371-376 (1997).

6. K. Sakai and H. Nishimura, "Asymmetric surrounding modulation in the determination of border-ownership," J. Cogn. Neurosci. 18, 562-579 (2006)

7. R. von der Heydt, T. Macuda, and F. T. Qiu, "Borderownership-dependent tilt aftereffect," J. Opt. Soc. Am. A 22, 2222-2229 (2005).

8. B. F. Manly, Randomization, Bootstrap and Monte Carlo Methods in Biology (Chapman \& Hall/CRC, 1997).

9. T. Hesterberg, S. Monaghan, D. S. Moore, A. Clipson, and R. Epstein, "Bootstrap methods and permutation tests," in The Practice of Business Statistics (Freeman, 2003), Chap. 18, pp. 39-41.

10. C. E. Connor, J. L. Gallant, D. C. Preddie, and D. C. Van Essen, "Responses in area V4 depend on the spatial relationship between stimulus and attention," J. Neurophysiol. 75, 1306-1308 (1996).

11. C. E. Connor, D. C. Preddie, J. L. Gallant, and D. C. Van Essen, "Spatial attention effects in macaque area V4," J. Neurosci. 17, 3201-3214 (1997).
12. T. Sugihara, F. T. Qiu, and R. von der Heydt, "Figureground organization and attention modulation in neurons of monkey area V2," J. Vision 4, 197a (2004).

13. H. Nishimura and K. Sakai, "Determination of borderownership based on the surround context of contrast," Neurocomputing 58-60, 843-848 (2004).

14. H. E. Jones, K. L. Grieve, W. Wang, and A. M. Sillito, "Surround suppression in primate V1," J. Neurophysiol. 86, 2011-2028 (2001).

15. M. Ito and H. Komatsu, "Representation of angles embedded within contour stimuli in area V2 of macaque monkeys," J. Neurosci. 24, 3313-3324 (2004).

16. K. Sakai and S. Tanaka, "Spatial pooling in the secondorder structure of cortical complex cells-computational analysis," Vision Res. 40, 855-871 (2000).

17. K. Sakai and Y. Hirai, "Neural grouping and geometric effect in the determination of apparent orientation," J. Opt. Soc. Am. A 19, 1049-1062 (2002).

18. B. S. Webb, N. T. Dhruv, S. G. Solomon, C. Tailby, and P. Lennie, "Early and late mechanisms of surround suppression in striate cortex of macaque," J. Neurosci. 25, 11666-11675 (2005).

19. C. Blakemore and F. W. Campbell, "On the existence of neurons in the human visual system selectively sensitive to the orientation and size of retinal images," J. Physiol (London) 203, 237-260 (1969).

20. P. Sajda and K. Baek, "Integration of form and motion within a generative model of visual cortex," Neural Networks 17, 809-821 (2004).

21. K. Baek and P. Sajda, "Inferring figure-ground using a recurrent integrate-and-fire neural circuit," IEEE Trans. Neural Syst. Rehabil. Eng. 13, 125-130 (2005).

22. Z. Li, "Border ownership from intracortical interactions in visual area V2," Neuron 47, 143-153 (2005). 